Chapter 8

\title{
Characterization of Magnetic Phases in Nanostructured Ferrites by Electron Spin Resonance
}

\author{
Rebeca Díaz-Pardo and Raúl Valenzuela \\ Additional information is available at the end of the chapter \\ http://dx.doi.org/10.5772/61508
}

\begin{abstract}
This chapter is dedicated to the analysis of the spin resonance response (ESR) of different magnetic phases, in nanoparticles (NPs) of magnetic oxides, or ferrites. Evidence of the correlations between resonance spectrum and magnetic structure has been published, of course, in many works; however, to our knowledge, it is somewhat scattered and not easily accessible. We have chosen to carry out this analysis mainly on ferrite NPs because these magnetic materials exhibit a wide variety of magnetic properties, and as a consequence, a large diversity of classic and novel applications in technological fields ranging from electronics to biomedics.
\end{abstract}

Keywords: EPR, FMR, ESR, Nanoparticles, Ferrites

\section{Introduction}

This chapter is dedicated to the analysis of the spin resonance response (ESR) of different magnetic phases, in nanoparticles (NPs) of magnetic oxides, or ferrites. Evidence of the correlations between resonance spectrum and magnetic structure has been published, of course, in many works; however, to our knowledge, it is somewhat scattered and not easily accessible. We have chosen to carry out this analysis mainly on ferrite NPs because these magnetic materials exhibit a wide variety of magnetic properties, and as a consequence, a large diversity of classic and novel applications in technological fields ranging from electronics to biomedics.

Ferrites can also be easily tailored, and in the nanometric range and at the appropriate temperatures, they exhibit one of the main magnetic structures: ordered ferrimagnetic, superparamagnetic, or paramagnetic. The latter structure is less complex, and electron 
paramagnetic resonance (EPR) has been known for $~ 50$ years, so in some way, this is a situation both classic and new. Ferrimagnetic and superparamagnetic NPs are more complex, and their resonance response is currently an active research subject.

We first describe the general basis of the classic resonance technique, where a microwave signal of constant frequency is applied on the sample, which is simultaneously subjected to a sweeping magnetic field, in order to achieve the resonance conditions. We tried to establish the main differences between the response of classic and metallic paramagnetic phases and the shapes of magnetically ordered (ferro- or ferrimagnetic) phases in the bulk state.

We then briefly review the novel properties associated with the nanometric size, followed by a short account of crystal structure and magnetic interactions in ferrites. EPR is briefly described, illustrated with some recent results. A basic description of the superparamagnetic phase is then given, with a review of some of the theoretical models proposed, as well as some of the most representative experimental results. The ferrimagnetic phase is then described with an accent on the differences originated by the exchange interactions, magnetocrystalline anisotropy, demagnetization fields, etc., and their effects on the response signal. The general response of magnetic nanoparticles in the ordered state is discussed. In the conclusion, finally, an attempt is made to establish a correlation between these phases and their resonance signals.

\subsection{Nanomagnetism}

In addition to the changes related to the decrease in scale down to the nanometric range, magnetic materials provide another source of novel properties. Many of the critical parameters in magnetism are found in the 1 to 200-nm scale of length, see Table 1 [1]. Two of the most interesting changes in magnetic structure as a consequence of the reduction in size are the change from multidomain to single domain and superparamagnetism.

Bulk samples of ferro- and ferrimagnetic materials are divided into magnetic domains (separated by domain walls) in order to decrease the magnetostatic energy, i.e., the energy associated with the presence of magnetic flux just outside the sample surface. Inside the magnetic domains exchange energy is at a minimum (all spins are parallel coupled) as well as magnetocrystalline energy (all spins are oriented into easy axes), and the magnetization of domains is oriented to provide a continuous magnetic flux inside the sample, thus avoiding any external flux. This is why most magnetic materials do not manifest any attraction or repulsion force (in the absence of an applied magnetic field). Magnetostatic energy is therefore eliminated, except for a small contribution from domain walls, where spins rotate from the orientation of a domain toward the orientation of the neighboring domain. There is also a small contribution to both exchange and anisotropy energy due to domain walls, as spins cannot be strictly parallel, neither oriented into easy directions within the domain wall. These contributions, however, are small as domain wall thickness is in the 10- to 100-nm range. Domain walls represent a very sensitive equilibrium in ferromagnetic materials. They can be displaced by very small applied, fields and their dynamics of propagation though defects (pinning sites) have a fundamental importance for soft magnetic materials [2]. 
As samples become smaller, there is a limit where it becomes more convenient, on an energetic basis, to eliminate the domain walls and form a single domain on the whole sample. This transition becomes feasible because magnetostatic energy is a function of the sample volume, while domain wall energy depends on their total surface. As a single domain, materials become magnetically harder; the coercive field typically exhibits an inverse relationship with the particle size going through a maximum for the transition between single domain and multidomain structure. Single-domain ferromagnets become harder because in the absence of domain wall, the main magnetization process is spin rotation, i.e., the applied magnetic field has to rotate each spin against the anisotropy. This is very different than having domain walls; as they are in a delicate equilibrium, a small field can easily displace them with large variation in oriented domains associated with their passage.

\begin{tabular}{ll}
\hline Property & Typical length range (nm) \\
\hline Exchange interaction & $0.1-1$ \\
\hline RKKY Interaction & $0.1-10$ \\
\hline Single-domain limit & $10-1000$ \\
\hline Superparamagnetic limit & $1-100$ \\
\hline Thickness of domain walls & $10-100$ \\
\hline
\end{tabular}

Table 1. Critical lengths in magnetism. Adapted from Guimaraes [1].

The reduction in scale can also lead to a superparamagnetic (SPM) phase. As discussed with more detail in Section 3, in an SPM phase, the thermal energy dominates over anisotropy, and the magnetization is therefore subjected to random fluctuations. It is interesting to note that in the SPM phase, the exchange interaction maintains the spin coupling up to the Curie temperature, which is higher than the temperature of transition from ordered to SPM phase, also known as blocking temperature. The magnetic properties in the SPM phase are different to both those in the ordered phase and in the paramagnetic phase. SPM properties allow many novel applications, particularly in the field of biomedicine.

\section{Ferrites}

Spinel ferrites are a large family of materials, with the structure of the natural spinel mineral, $\mathrm{MgAl}_{2} \mathrm{O}_{4}$, first determined by Bragg [3]. The spinel is a very stable crystal structure; it is almost enough to satisfy the conditions of neutral electric charge, relatively small cation radii, and a $3 / 4$ cation-to-anion ratio [2]. These conditions allow several cation combinations such as 2,3 (as in $\mathrm{Ni}^{2+} \mathrm{Fe}^{3+}{ }_{2} \mathrm{O}_{4}$ ), 2,4 (as in $\mathrm{Co}_{2} \mathrm{GeO}_{4}$ ), 1,3,4 (as in $\mathrm{LiFeTiO}_{4}$ ), 1,3 (as in $\mathrm{Li}_{0.5} \mathrm{Fe}_{2.5} \mathrm{O}_{4}$ ), 1,2,5 (as in $\mathrm{LiNiVO}_{4}$ ), and 1,6 (as in $\mathrm{Na}_{2} \mathrm{WO}_{4}$ ). Most of ferrites with significant magnetic properties are of the 2,3 type and contain $\mathrm{Fe}^{3+}$. An important ferrite is magnetite $\mathrm{Fe}^{2+} \mathrm{Fe}^{3+}{ }_{2} \mathrm{O}_{4}$, also referred as $\mathrm{Fe}_{3} \mathrm{O}_{4}$, which is the oldest known magnetic material. The name "magnetic" is derived from 
Magnesia, which is the region where magnetite was first discovered in Greece in the 6th century BC. It is interesting that magnetite, the magnetic material first reported in history, is currently an extremely active field in basic and applied research. This is certainly due to its fascinating magnetic and electric properties, associated with the coexistence of ferrous and ferric cations in equivalent crystal sites. A related magnetic material also particularly interesting is maghemite (or $\gamma-\mathrm{Fe}_{2} \mathrm{O}_{3}$ ), which possesses only $\mathrm{Fe}^{3+}$ and the same spinel structure often obtained by oxidation of magnetite. Since the cation to anion ratio is not $3 / 4$, it contains vacancies in a fraction of cation sites; by using the spinel formula, it can be represented as $\square_{1 / 3} \mathrm{Fe}^{3+}{ }_{8 / 3} \mathrm{O}_{4}$. Here, $\square$ stands for vacancies.

The crystal symmetry can be understood by considering a face centered cubic (fcc) lattice of oxygen, leading to two kinds of cation sites: 64 tetrahedral sites and 32 octahedral sites for a unit cell, which is formed by 8 times the basic formula $\mathrm{D}^{2+} \mathrm{T}^{3+}{ }_{2} \mathrm{O}_{4}\left(\mathrm{D}^{2+}\right.$ stands for a divalent cation, and $\mathrm{T}^{3+}$ for a trivalent cation). Only one-eighth of tetrahedral sites is occupied and half of octahedral sites as well. The space group is $\mathrm{Fd} 3 \mathrm{~m}$. This structure is shown in Figure 1.

In the spinel mineral $\mathrm{MgAl}_{2} \mathrm{O}_{4}, \mathrm{Mg}^{2+}$ cations occupy tetrahedral sites and $\mathrm{Al}^{3+}$ cations are found in octahedral sites, which appears as a stable arrangement, as far as divalent cations are surrounded by less anions (four anions in the tetrahedral site) than trivalent cations with higher electric charge and enclosed by 6 anions. This structure is known as the normal spinel, and is indicated by using the system $\left(\mathrm{D}^{2+}\right)\left[\mathrm{T}^{3+}{ }_{2}\right] \mathrm{O}_{4}$. Parenthesis indicates occupancy of tetrahedral sites, also known as " $\mathrm{A}$ " sites, and square brackets show cations on octahedral sites or " $\mathrm{B}$ " sites. A different cations distribution is $\left(\mathrm{T}^{3+}\right)\left[\mathrm{T}^{3+} \mathrm{D}^{2+}\right) \mathrm{O}_{4}$, where the divalent cation goes to an octahedral site and trivalent cations are found both on tetra and octahedral sites. This is the inverse spinel. An intermediate cation distribution has also been observed for some ferrites, $\left(D_{1-\delta} T_{\delta}\right)\left[D_{\delta} T_{2-\delta}\right] O_{4}$, where $\delta$ is the degree of inversion.

The cation distribution in spinels was a problem for some time, but it is now well understood. The involved energies are the elastic energy, associated with the lattice deformation produced by cation radii differences. The electrostatic energy, also known as the Madelung energy, which depends on the overall electric charge distribution; divalent cations on small sites and trivalent cations on larger sites should stabilize the spinel. The crystal field energy has also a large influence on cation site "preference," and it is related mainly to the geometry of $d$-orbitals (or electronic orbitals for nontransition cations). $d^{5}$ orbitals, for instance, can occupy both types of sites as these orbitals (in high-spin state) have spherical geometry (see Table 2).

\begin{tabular}{ccc}
\hline Tetrahedral & Octahedral & Undistinguished \\
\hline $\mathrm{Cd}^{2+}$ & $\mathrm{Co}^{2+}$ & $\mathrm{Fe}^{3+}$ \\
\hline $\mathrm{Zn}^{2+}$ & $\mathrm{Ni}^{2+}$ & $\mathrm{Mn}^{2+}$ \\
\hline & $\mathrm{Cu}^{2+}$ & $\mathrm{Mg}^{2+}$ \\
\hline & $\mathrm{Fe}^{2+}$ & $\mathrm{Mo}^{2+}$ \\
\hline & $\mathrm{Li}^{1+}$ & \\
\hline
\end{tabular}

Table 2. Cation preferences for spinel sites. Adapted from [2]. 

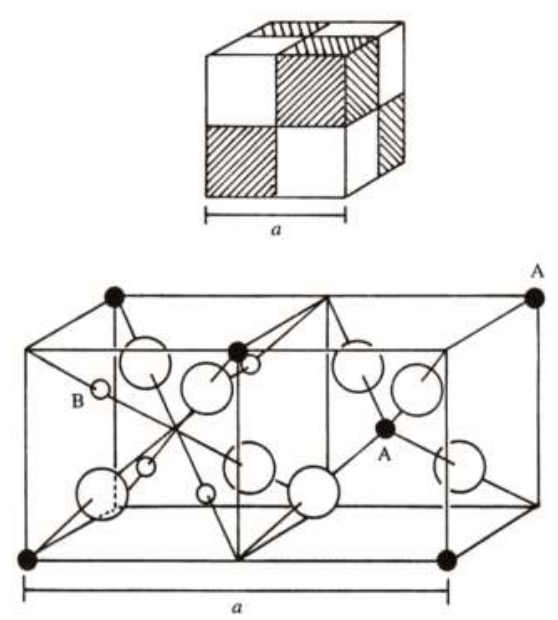

Figure 1. Representation of the spinel structure. The unit cell can be divided into octants (above). The detailed structure of two octants is illustrated below. Large spheres are oxygen, small black spheres indicate cations in tetrahedral sites, and small white spheres represent cations in octahedral sites [2].

An outstanding characteristic of the spinel structure is that it admits an extremely large variety of total solid solutions. The divalent cations in the spinel formula can be formed by a combination of two (or more) cations; $\mathrm{Fe}^{3+}$ can also by partially or totally substituted by another trivalent cation, always maintaining the spinel crystal structure. A very well studied system (or "family") is $\mathrm{Zn}-\mathrm{Ni}$ ferrites with the general formula $\mathrm{Zn}_{x} \mathrm{Ni}_{1-x} \mathrm{Fe}_{2} \mathrm{O}_{4}$, with $0 \leq x \leq 1$. The end compositions are nickel ferrite, $\mathrm{NiFe}_{2} \mathrm{O}_{4}$ (for $x=0$ ), and zinc ferrite, $\mathrm{ZnFe}_{2} \mathrm{O}_{4}(x=1)$. Nickel ferrite is an inverse spinel, while zinc ferrite is a normal one; due to the features of magnetic interactions in spinel ferrites, nickel ferrite is ferrimagnetic with a Curie point about $858 \mathrm{~K}$, while zinc ferrite is antiferromagnetic with a Néel temperature about $9 \mathrm{~K}$. The properties of this family of compounds can be "tailored" between these two extreme behaviors just by varying the chemical composition.

The magnetic structure and interactions in ferrites can be understood on the basis of superexchange interactions between two transition cations separated by an oxygen, as shown in Figure 2 . The electron spin up in the $p$-orbital of the oxygen can occupy for a short period of time the empty half of the $d$-orbital in the transition cation at right, if the occupied state in this cation is spin down. As the $p$-orbital of oxygen exhibits, for the same given period of time, an available site for an electron with spin up, the electron of the transition cation on the left side of the oxygen can occupy this place, if its spin is pointing up. This mechanism leads therefore to an antiparallel arrangement of spins on the transition cations. In spinels, there are two sublattices, a tetrahedral one (with one cation per formula) and an octahedral one (with two cations per formula). In most cases, a resultant appears and most ferrites possess a net magnetization. The $\mathrm{Zn}-\mathrm{Ni}$ ferrite family is a very good example of the richness of magnetic properties and structure of ferrites. 


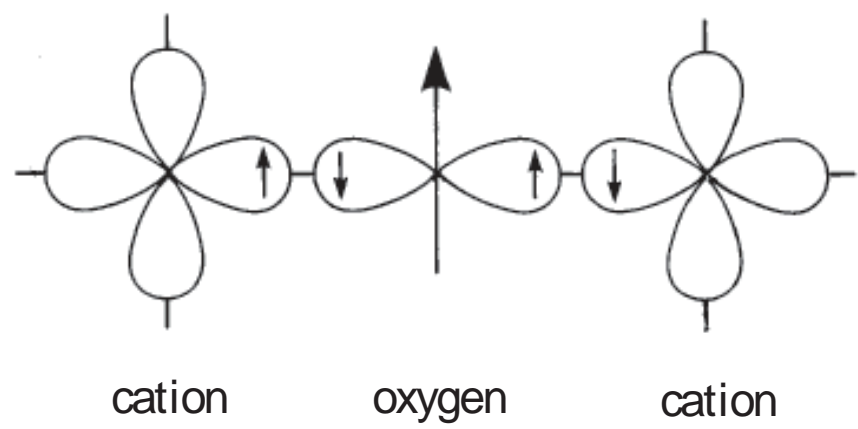

Figure 2. Superexchange interactions in ferrites.

The magnetic interactions in spinel ferrites can be easily understood by considering two B sites and one A site about an oxygen, see Figure 3. The center and right side of this figure, where the large circle represents the oxygen ion, the small circle above it stands for the cation on the A site, and the two small circles below represent B sites. Note that this arrangement is 3D, but for this schematic illustration, it has been simplified on a plane. Nickel ferrite is an inverse spinel, $\mathrm{Ni}^{2+}$ goes to $\mathrm{B}$ sites, one ferric ion occupies the other $\mathrm{B}$ site, and the other $\mathrm{Fe}^{3+}$ is located on the A site. All magnetic interactions between cations are antiparallel (superexchange).
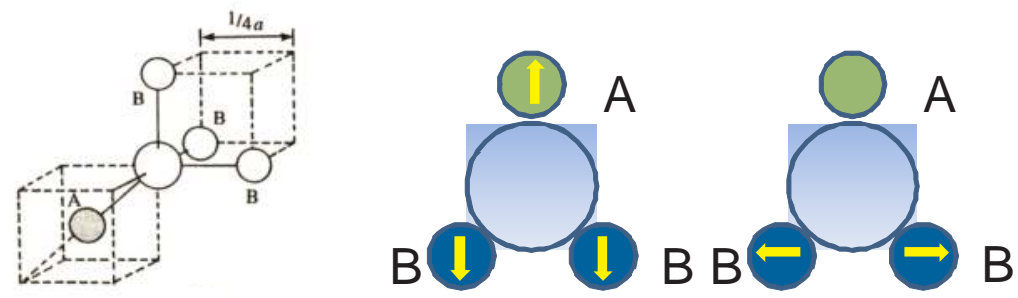

Figure 3. Schematic spin arrangements in spinel ferrites. Left: a section of the crystal structure showing the cation occupancy about an oxygen. Center: the ferrimagnetic spin orientation of the crystal sites for nickel ferrite. Right: the antiferromagnetic structure for the zinc ferrite.

There are A-O-B interactions between the cation on A site and the cations on B sites, and an $\mathrm{B}-\mathrm{O}-\mathrm{B}$ interaction between the cations on the two B sites. However, due to the axial symmetry of oxygen $p$-orbitals, superexchange interaction becomes more efficient when cations and oxygen are in an axial arrangement. This makes a strong difference between interactions; A$\mathrm{O}-\mathrm{B}$ interaction is far more efficient than B-O-B interactions since the latter has a $90^{\circ}$ angle, while the former is closer to $180^{\circ}$. As a result, both spins on B sites are antiparallel to the A spin. The magnetic moment of $\mathrm{Fe}^{3+}$ on $\mathrm{A}$ site cancels with the magnetic moment of $\mathrm{Fe}^{3+}$ on $\mathrm{B}$ site, and the resulting magnetic moment is the spin value for nickel $\mathrm{Ni}^{2+}$ (2.3 Bohr magnetons), as shown in Figure 3 center. This is a strong interaction; the Curie transition is about $858 \mathrm{~K}$. 
In the case of zinc ferrite, iron ions occupy the B sites and zinc is located on the A site. Since $\mathrm{Zn}^{2+}$ is a $3 d^{10}$ ion, it has no magnetic moment, and the only interaction is $\mathrm{B}-\mathrm{O}-\mathrm{B}$, leading to an antiparallel arrangement of spins (Figure, 3 right). The spins are identical, and the material becomes antiferromagnetic. The Néel temperature is about $9 \mathrm{~K}$, and this clearly illustrates the weakness of this superexchange interaction, as compared with $\mathrm{AOB}$ of the nickel ferrite case. Based on transition temperatures, A-O-B interaction is roughly 95 times stronger than $\mathrm{B}-\mathrm{O}-$ B interaction.

\section{Electron paramagnetic resonance}

Electron paramagnetic resonance (EPR) or electron spin resonance (ESR) is a technique of observing resonance absorption of microwave power by unpaired electron spins aligned with a magnetic field [4]. The electron paramagnetic resonance (EPR) spectroscopy is similar to the nuclear magnetic resonance (NMR) except for the fact that EPR arises from the interaction between unpaired electrons and electromagnetic radiation.

The history of EPR can be told beginning with the Stern-Gerlach experiment in 1921. They observed that a beam of silver atoms split into two lines when it was subjected to a magnetic field. In contrast with the line splitting in optics found by Zeeman in 1896, these phenomena could not be explained by the angular momentum of the electrons. At the time quantum mechanics was still developing and was still an emerging field. Three more years passed before Uhlenbeck and Goudsmit found an explanation for these anomalous Zeeman effect when they postulated the spin $[5,6]$.

The development of the EPR was furthered by the World War II since after the war was over, there was available a great amount of microwave instruments from the radar equipment used in the war. It was in 1945 when a Russian physicist, Zavoisky, observed the first EPR spectrum. $\mathrm{He}$ observed a radiofrequency absorption line from a $\mathrm{CuCl}_{2} \cdot 2 \mathrm{H}_{2} \mathrm{O}$ sample. One year later, the first NMR spectrum was observed and through the first decade both techniques developed simultaneously. Nevertheless, EPR spectroscopy had a few challenges from lack of microwave components and limited microwave power to expensive instrumentation and consequently was left behind by NMR for a couple of decades [4-6].

It was until the 1980s that the instrumentation became cheaper and more manageable and the first pulse EPR was released to the market. A decade later the high field spectrometer was released and since then the interest in EPR as a characterization technique has considerably increased.

The spin angular momentum $S$ gives rise to a magnetic moment $\mu=-\mathrm{g} \mu_{\mathrm{B}} S$, where $g$ is the $\mathrm{g}$ factor; its value for free electrons is $g=2.0023193043617$ and $\mu_{\mathrm{B}}$ is the Bohr magneton. When the electrons are subjected to an external field $H$ (it is customary to place the field along the $z$ axis), the energy levels of the degenerate spin states split depending on their quantum magnetic moment $m_{\mathrm{s}}= \pm 1 / 2$ and the strength of the magnetic field as shown in Figure 4 [4-6]. 


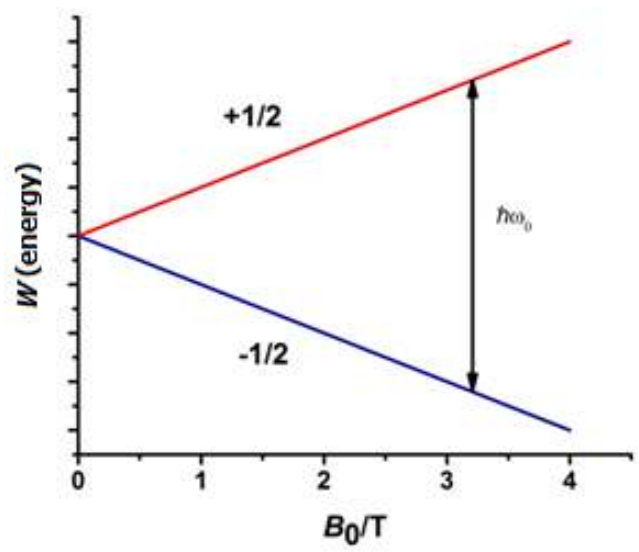

Figure 4. Splitting of the energy levels of an electron spin in a magnetic field at the resonance frequencies.

When the irradiation at a given frequency $\omega_{0}$ is the same as the energy difference $\Delta E$ between both states, a resonant absorption takes place. This frequency is named the Larmor frequency $\omega_{0}=-\gamma H_{0}$ after J. Larmor [6]:

$$
\Delta W=\hbar \omega_{0}=g \mu_{B} H
$$

The detection of that absorption is the most important principle of this spectroscopy.

For temperatures above the Curie temperature, the magnetic moments in an external field obey the Bloch equations. These equations follow the Larmor theorem where the motion of the magnetic moments in a magnetic field originates a torque [6-9]:

$$
\hbar \frac{d \mathbf{M}}{d t}=\mathbf{M} \times \gamma \mathbf{H}
$$

To detect the absorption, there is an oscillating microwave field $\mathbf{H}_{1}=\left(H_{x}, H_{y}, 0\right)=\left(H_{1} \cos \left(\omega_{\mathrm{mw}} t\right)\right.$, $\left.H_{1} \sin \left(\omega_{\mathrm{mw}} t\right), 0\right)$ aside from the external magnetic field along the $z$ axis. This magnetic field deviates the magnetization away from its equilibrium position. To describe accurately the motion of the magnetization vector, it is important to take into account the relaxation effects. The relaxation time has two components, the longitudinal relaxation time, which is the average time in which the magnetization vector returns to its thermal equilibrium state, and the transverse relaxation time, which characterizes the loss of coherence in the transverse plane due to spin interactions [5-8].

The evolution of the magnetization as a function of time and its dependence of the magnetic field is given by the equation motion derived by F. Bloch [5-6]: 


$$
\hbar \frac{d \mathbf{M}}{d t}=\mathbf{M}(t) \times \gamma \mathbf{H}(t)-\mathbf{R}\left(\mathbf{M}(t)-\mathbf{M}_{0}\right)
$$

where $\gamma$ is the gyromagnetic ratio, $\mathbf{H}=\left(H_{1} \cos \left(\omega_{\mathrm{mw}} t\right), H_{1} \sin \left(\omega_{\mathrm{mw}} t\right), H_{z}\right)$, and $\mathbf{R}$ is the relaxation tensor given by [6]:

$$
\mathbf{R}=\left(\begin{array}{ccc}
1 / T_{2} & 0 & 0 \\
0 & 1 / T_{2} & 0 \\
0 & 0 & 1 / T_{1}
\end{array}\right)
$$

Relaxation phenomena need to be taken into account to describe the motion of the magnetization vector. $T_{1}$ is the longitudinal relaxation time; this parameter characterizes the process that makes the magnetization vector return to its thermal equilibrium. $T_{2}$ is the transverse relaxation time which describes the loss of coherence in the transverse plane due to spin-spin interactions.

Electron paramagnetic resonance is a very useful technique to study the properties of bulk paramagnetic compounds including their transitions to the magnetic ordered state. Below this temperature, ferromagnetic resonance (FMR) or antiferromagnetic resonance (AFMR) are detected. EPR in paramagnetic samples give information such as the resonance active ion valence and the symmetry of the ligand environment. In the case of nanoparticles, the theory of EPR is not quite the same as in bulk; however, a study of the thermal variations of EPR spectra can be very informative because of the high sensibility of the EPR technique [10].

Let's recall that magnetic resonance is observable only in materials that contain a sufficient number of permanent magnetic dipoles; if the origin of these dipoles is electronic, then the resonance is detectable with a population even fewer than $10^{11}$ dipoles [8].

The first paramagnetic resonance absorption in metals due to the conduction electrons was observed by Feher and Kip [8]. The conduction electrons have an effect on the shape and intensity of the resonance lines. However, until the theoretical study of Dyson, there were not many studies in conduction electron paramagnetic resonance in metals [11].

In a metal, the electrons are assumed to diffuse as free particles and the magnetic moments of each of them can be seen as free-particle moments. When the metal is placed in a radiofrequency electromagnetic field and at the same time in a perpendicular uniform magnetic field, a certain macroscopic magnetization is created as a result of the magnetic moments of the conduction electrons. The penetration of the radio frequency field into the metal is modified by the magnetization. Actually, only the layers near the surface contribute, since the excitation field $H_{1}$ penetrates only a small depth into the metal. This is called the skin effect [11]. The magnetization $M$ shows a resonant behavior and becomes large when the frequency of the field is nearly equal to the resonant frequency. The absorption field observed is a measure of the total energy absorbed in the metal both by eddy currents and by the resistive out of phase component of the magnetization. 
There are four assumptions which need to be taken into account to study the dependence of the line width $[8,11]$ :

1. The electrons which carry the magnetization in a metal are assumed to lie at the top of the Fermi distribution of the conduction electrons and to move with constant velocity.

2. Each electron moves as an independent classical particle with random changes in their direction.

3. The spin of each particle is a quantum independent variable. It is unaffected by collisions and only the local magnetic field has an effect on them.

4. Given any time interval $t$ and $U$, a volume relaxation time there is a probability $\sim \exp (-t / U)$ that the spin state of an electron will not be randomly distributed by collisions during this interval.

These assumptions give as a result a finite line width proportional to $1 / U$ in the resonance signal from nondiffusing electrons. The penetration of the radio frequency field into the metal will be limited by the skin depth, which is given by [11]

$$
\delta=\left(c^{2} / 2 \pi \sigma \omega\right)^{1 / 2}
$$

where $c$ is the speed of light, $\sigma$ is the conductivity of the metal, and $\omega$ is the radio frequency. If the skin depth is larger than the main free path, we are in the domain of the classical skin effects; if it is equal to the main free path, then we are in the anomalous case. In fact, unlike paramagnetic insulators, in which the varying part of the magnetization depends on the magnitude of the applied oscillating field, in metals, there is also a dependence of the local values of the oscillating field on the magnetization because of the skin depth. A typical spectrum (metallic $\mathrm{Na}$ ) is shown in Figure 5.

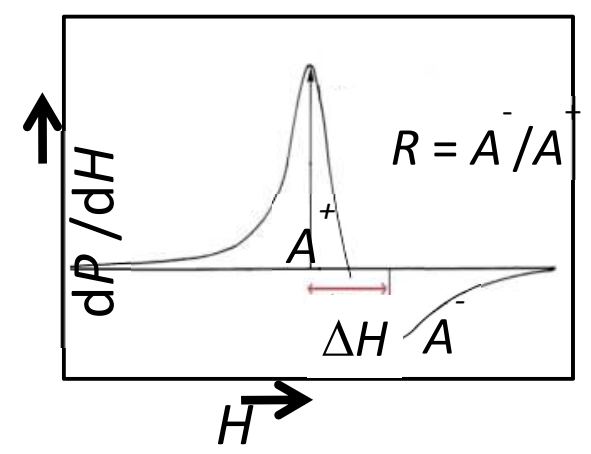

Figure 5. Typical first derivative EPR spectrum observed in colloidal samples of $\mathrm{Na}$, with mean diameter particle small compared to the skin depth. The basic parameters and the definition of $R$ are indicated. Adapted from Feher and Kip [8]. 
The magnetic susceptibility in metals has a diamagnetic component due to the circulation of electrons in the field $H$. This is opposed by the normal paramagnetic component due to unpaired electrons. This is related with another EPR parameter that has been studied in metals: the $g$ factor [8,12]. The relation between the resonance values of the constant magnetic field $H_{0}$ and the frequency is determined by the magnitude of the $g$ factor of the conduction electrons. The existence of internal interactions in a metal leads to a shift in the value of the $g$ factor.

Experimentally, the $g$ factor has been measured in different paramagnetic metals giving rise to values very close to the $g$ free electron value $g=2.0023$ as shown in Table 3 . This is a measure of the spin-orbit coupling. It has been possible to study $S$-state ions in metals in order to understand the interaction between the conduction electrons and the inserted ions.

\begin{tabular}{ll}
\hline Metal & Experimental values $\Delta g=g-2.0023$ \\
\hline $\mathrm{Li}$ & $\Delta g<10^{-4}$ \\
\hline $\mathrm{Na}$ & $\Delta g=-(8 \pm 2) \times 10^{-4}$ \\
\hline $\mathrm{Be}$ & $\Delta g=+(9 \pm 2) \times 10^{-4}$ \\
\hline
\end{tabular}

Table 3. Experimental values for the $g$ shift. Adapted from Feher and Kip [8].

EPR spectroscopy has made contributions in understanding the bonding and the electronic structure of molecular species with metal-metal bonds. Some of the information obtained through this technique includes determining whether unpaired electrons reside in metal-based or ligand-based molecular orbitals, giving information of the metal center's total electronic spin, which also provides information of its oxidation state, broadly have information of the distribution of the unpaired electrons between metals and organic ligands [13].

\subsection{Magnetic nanoparticles}

Iron-based oxide nanoparticles materials have been widely studied because of their technological importance. $\gamma-\mathrm{Fe}_{2} \mathrm{O}_{3}$ is a very popular material for magnetic tape purposes. Another popular material in high density recording media is $\mathrm{BaFe}_{12} \mathrm{O}_{19}$ and its substituted derivatives since it can be doped with other cations in order to reduce their magnetocrystalline anisotropy [10].

Bulk $\mathrm{Fe}_{2} \mathrm{O}_{3}$ exists as a ferrimagnetic $\gamma-\mathrm{Fe}_{2} \mathrm{O}_{3}$ (maghemite) or antiferromagnetic $\alpha$ - $\mathrm{Fe}_{2} \mathrm{O}_{3}$ (hematite). Maghemite nanoparticles are a representative model for the experimental study of nanoparticles and for testing theoretical concepts. In contrast, bulk hematite is antiferromagnetic below the Morin temperature and weakly ferromagnetic above this temperature [10].

At room temperature, the EPR of $\gamma-\mathrm{Fe}_{2} \mathrm{O}_{3}$ and $\mathrm{BaFe}_{2} \mathrm{O}_{4}$ and nanoparticles show a tow line pattern as shown in Figure 6 [9], which is typical of superparamagnetic resonance in contrast with the $\mathrm{BaFe}_{12} \mathrm{O}_{19}$ where the narrow line is more pronounced (as shown in Figure 6c). In these sample, the line width $\Delta H \sim 120$ Oe and $g=2.00$; this value of the $g$ factor is typical paramagnetic. 


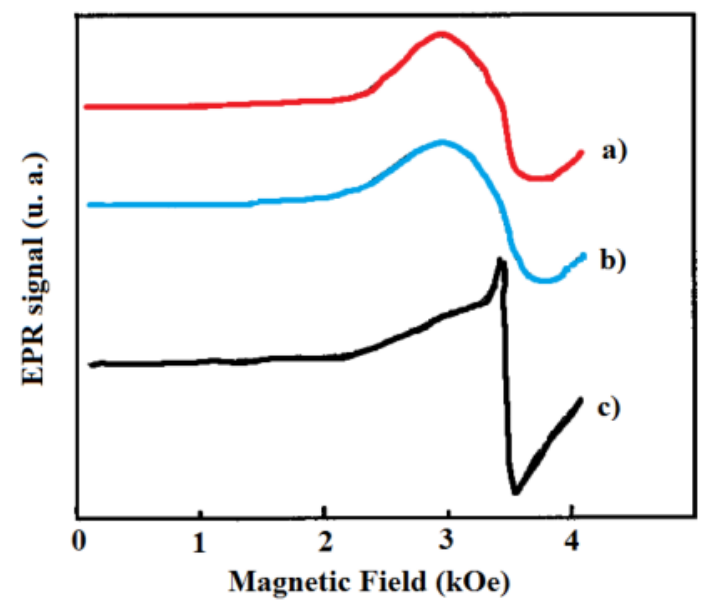

Figure 6. Room temperature EPR spectra of nanoparticles: (a) $\mathrm{Fe}_{2} \mathrm{O}_{3}$, (b) $\mathrm{BaFe}_{2} \mathrm{O}_{4}$, and (c) $\mathrm{BaFe}_{12} \mathrm{O}_{19}$. Adapted from Koksharob et al. [10].

It is shown in Figure 7a that below $100 \mathrm{~K}$, the broad line 1 shows a typical superparamagnetic (SPM) behavior of single-domain particles in the absence of transitions to a magnetic ordered state. SPM behavior will be discussed in the following section. Below $50 \mathrm{~K}$, new resonances appear such as 2 and 3. Point 2 is a typical paramagnetic resonance signal. On the other hand, point 3 is characteristic of a phase transition $\alpha-\mathrm{Fe}_{2} \mathrm{O}_{3}$ of the hematite to antiferromagnetic phase.
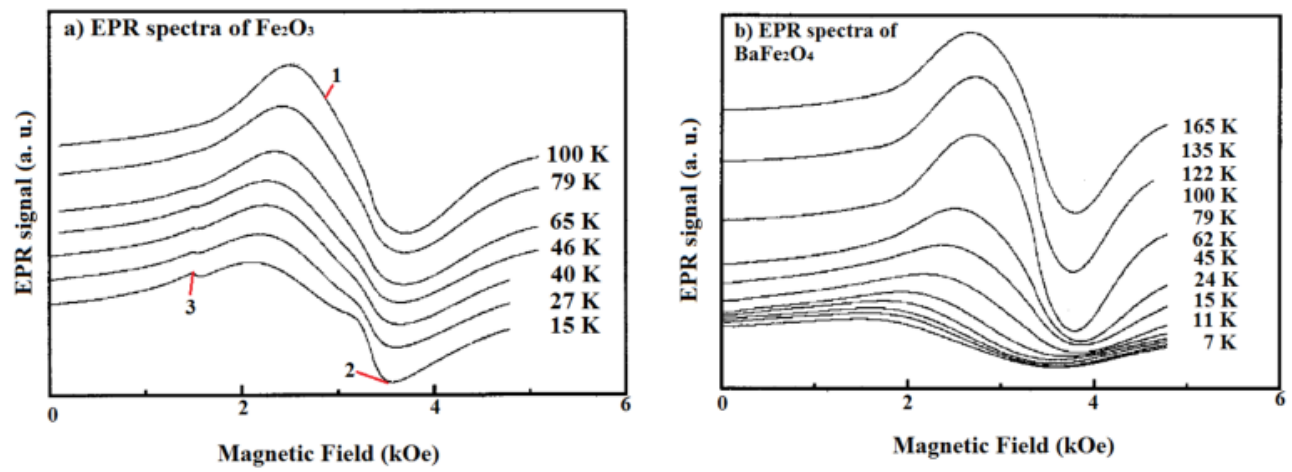

Figure 7. (a) EPR spectra of $\mathrm{Fe}_{2} \mathrm{O}_{3}$ nanoparticles; (b) EPR spectra of $\mathrm{BaFe}_{2} \mathrm{O}_{4}$ nanoparticles. Adapted from Koksharob et al. [10].

The bulk thermodynamics phase diagram [14] shows several interchangeable iron oxide phases. For nanoparticles, the mutual phase transition should be easier. For this reason, it is accurate to expect a multiphase composition in $\mathrm{Fe}_{2} \mathrm{O}_{3}$ nanoparticles. In point 3 , the value $g$ is 4.03 , which is characteristic of a high spin state $\mathrm{Fe}^{3+}$ in the rhombic crystal field. The antifer- 
romagnetic transition is associated with the $\alpha-\mathrm{Fe}_{2} \mathrm{O}_{3}$ phase. In rhombohedral $\alpha-\mathrm{Fe}_{2} \mathrm{O}_{3}$ as well as in $\gamma-\mathrm{Fe}_{2} \mathrm{O}_{3}$, there is a departure from axial symmetry. The paramagnetic signal in point 2 is attributed to the $\gamma-\mathrm{Fe}_{2} \mathrm{O}_{3}$ phase; it has a $g$ value of 2.02, and it is due to the octahedral symmetry sites of $\mathrm{Fe}^{3+}$ in the spinel structure.

Bulk $\mathrm{BaFe}_{2} \mathrm{O}_{4}$ is nonferrimagnetic. On the contrary, $\mathrm{BaFe}_{2} \mathrm{O}_{4}$ demonstrate an EPR anomaly near $125 \mathrm{~K}$ that could indicate the presence of a $\mathrm{Fe}_{2} \mathrm{O}_{3}$ phase. On the other hand, bulk hexaferrite $\mathrm{BaFe}_{12} \mathrm{O}_{19}$ is a representative case of FMR because of its very high uniaxial anisotropy. However, in the nanoparticles, the contribution of the particle's surface is appreciable and can reduce the total anisotropy energy as well as crystalline defects due to stress and strain. The EPR spectra reveal the effect of superparamagnetic fluctuations narrowing the resonance [10] and will be discussed in the next section.

\section{Spin resonance in superparamagnetic phases}

The superparamagnetic (SPM) state is characterized by random fluctuations of the magnetization due to thermal excitation. It appears in ferro- and ferrimagnetic particles, which are sufficiently small and thus single domain, typically in the nanometric range $(1-100 \mathrm{~nm})$. It is important to note that in contrast with the paramagnetic state, characterized by random fluctuations of individual, noninteracting spins in the SPM phase, these fluctuations involve the whole magnetization vector, which is the sum of all individual moments of the particle. As SPM occurs at temperatures below the Curie transition, exchange interaction is effective, and spins are therefore coupled. It is by this reason that some authors describe SPM as "macro-spin" fluctuations.

A simple approach to SPM can be obtained by considering the magnetocrystalline anisotropy energy, $E_{\mathrm{K}}=K V$, for a nanoparticle of volume $V$ and anisotropy constant $K$. When this product becomes small and comparable to the thermal energy $E_{\mathrm{T}}=k_{\mathrm{B}} T$ (where $k_{\mathrm{B}}$ is the Boltzmann constant), the magnetization oscillates by thermal excitations and easy directions vanish. A simplified graphical model is shown in Figure 8. A uniaxial nanoparticle has two easy magnetization directions, i.e., two magnetization orientations that lead to a minimum in anisotropy energy. These orientations are separated by an energy barrier $E_{\mathrm{K}}=K V$. Superparamagnetism appears when the thermal energy becomes comparable to anisotropy energy, and therefore the energy barrier is dominated by thermal oscillations.

A nanoparticle of a given composition and constant volume typically shows a ferro/ferrimagnetic behavior at low temperatures and can become superparamagnetic as $T$ increases. At low temperatures, $K$ is large and $T$ is small, and the opposite is true as $T$ increases. The temperature at which SPM occurs is known as the blocking temperature, $T_{B}$.

The probability of reversal of magnetization is given by the Néel-Arrhenius or Néel-Brown relation $[15,16]$ : 


$$
\tau_{\mathrm{m}}=\tau_{0} \exp \left(K V / k_{\mathrm{B}} T\right)
$$

where $V$ is the volume of the nanoparticle, $K$ is the anisotropy constant, $T$ is the temperature, $\tau_{\mathrm{m}}$ is the average length of time for a magnetization reversal to occur, and $\tau_{0}$ is a characteristic time constant related with the gyromagnetic precession, known also as the attempt time for a given material. Typical values are in the $10^{-9}$ to $10^{-10} \mathrm{~s}$ range. Under an applied magnetic field, the blocking temperature variations can be expressed (for uniaxial materials) as [17]

$$
T_{\mathrm{B}}(H)=\frac{K V}{k_{\mathrm{B}} \ln \left(\tau_{\mathrm{m}} / \tau_{0}\right)}\left[I-\left(\frac{H}{H_{k}}\right)\right]^{2} .
$$

where $H$ is the applied field, $H_{\mathrm{K}}$ is the anisotropy field, and $a$ has a typical value of 1.5 [18].

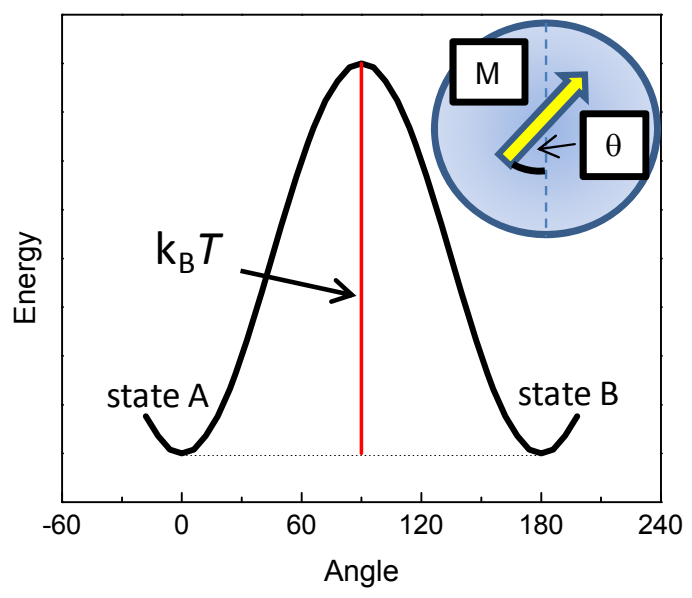

Figure 8. Schematics of the energy in a single-domain particle (with uniaxial anisotropy) as a function of the angle between easy axes and the magnetization direction. For SPM nanoparticles, the energy barrier is comparable to the thermal energy, thus resulting in magnetization random oscillations.

It is interesting to note that the blocking temperature, $T_{\mathrm{B}}$, depends on the time window, $t_{\mathrm{w}}$ of the particular experimental technique. $t_{\mathrm{w}}$ is the effective length of time during the measurement. If $t_{\mathrm{w}} \gg \tau_{\mathrm{m}}$ the magnetization will show several reversals and the state of the particle will we taken as SPM. On the contrary, if $t_{\mathrm{w}}<<\tau_{\mathrm{m}}$, magnetization will exhibit a stable state, and therefore the material will be considered as in the ordered phase. The time window for $T_{\mathrm{B}}$ determination from magnetization measurements in the ZFC-FC technique (in a SQUID machine) is about $10^{-1} \mathrm{~s}$, while for Mössbauer spectroscopy or spin resonance techniques $t_{\mathrm{w}}$ is about $10^{-6} \mathrm{~s}$. A model to describe the crossover from the SPM state to the blocked phase in magnetic nanoparticles has been recently proposed [19]. It is based on the Stoner-Wohlfarth 
model and also assumes noninteracting nanoparticles with uniaxial anisotropy and uniform random reversal of magnetization.

SPM materials show no hysteresis in $M$ vs. $H$ plots, i.e., absence of both coercive field and remanent magnetization, as illustrated in Figure 9. For an ensemble of noninteracting particles at temperatures low enough $\left(T_{\mathrm{B}}<\mathrm{T}<K V / 10 k_{\mathrm{B}}\right)$, a simple model for the magnetization behavior, based on the paramagnetic theory, is

$$
M(H) \approx n \mu \tanh \left(\frac{\mu_{0} H \mu}{k_{B} T}\right)
$$

At higher temperatures $\left(T>K V / k_{\mathrm{B}}\right)$,

$$
M(H) \approx n \mu L\left(\frac{\mu_{0} H \mu}{k_{B} T}\right)
$$

where $n$ is the density (atoms/volume), $\mu$ is the magnetic moment (the "macrospin"), $M$ is the magnetization, and $L$ is the Langevin function, $L(x)=1 / \tanh (x)-1 / x$, with $x=\mu_{0} H \mu / T k_{\mathrm{B}}$. This behavior is very convenient for many applications, especially in biomedicine, where magnetic nanoparticles manifest a magnetization only when subjected to a magnetic field, thus avoiding interparticle interactions (which could lead to aggregation) at $H=0$. In contrast, SPM is useless for magnetic recording since the absence of a remanent state eliminates any possibility of memory.

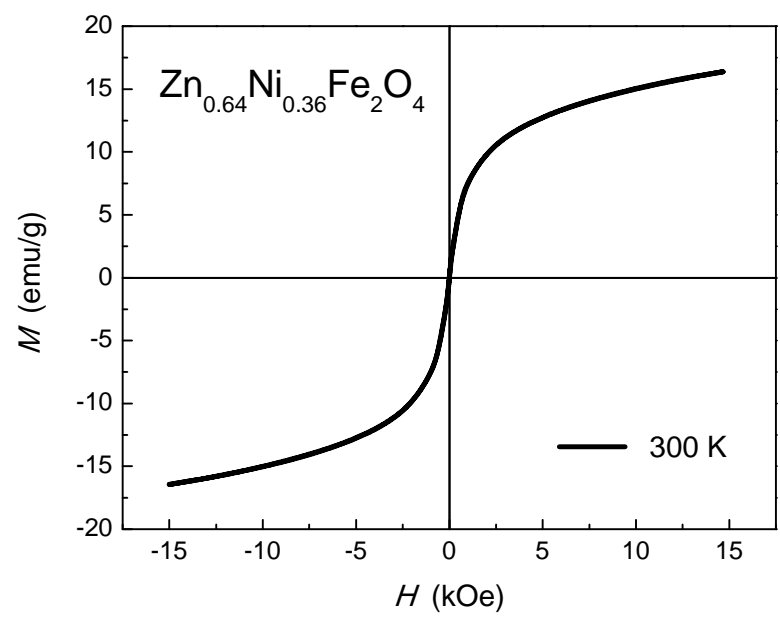

Figure 9. Hysteresis loop of $\mathrm{ZnNi}$ nanoparticles in the superparamagnetic phase [20]. 
The kinetic approach [21,22] is known with this name because it makes use of the FokkerPlanck-type equation used by Brown [16] to analyze viscosity problems. The main criticism for this approach is that it predicts an increase of the line width with temperature, which is opposite to the large majority of experimental results. On some cases, a tendency to show a constant line width has been observed, but to our knowledge, no significant increase in $\Delta H$ has been reported in SPM phases as $T$ increases.

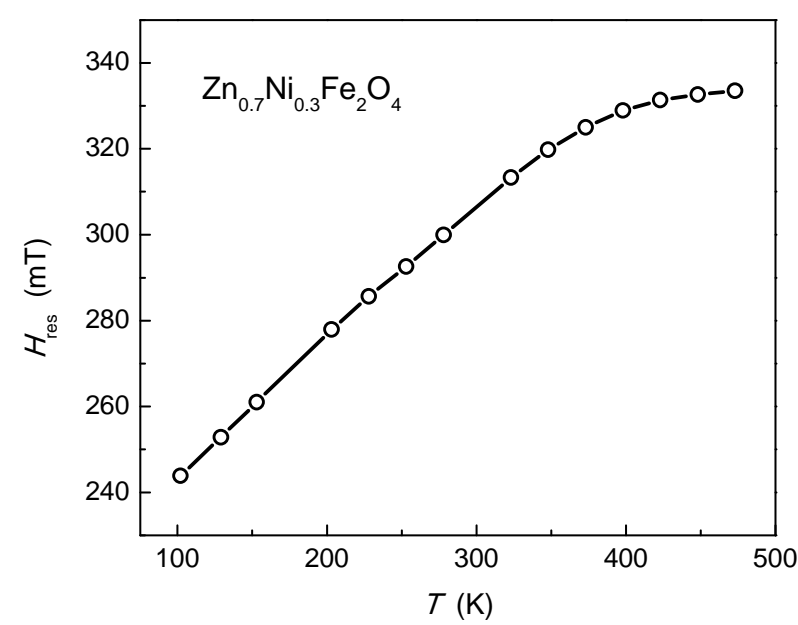

Figure 10. Resonance field as a function of temperature for $\mathrm{Zn}-\mathrm{Ni}$ ferrite NPs, at $f=9.45 \mathrm{GHz}$ [23].

The SPM state has elicited the proposal of a number of theoretical models to account for its magnetization, and more specifically to understand the features of the resonance spectrum. Some models [24, 25] discuss the longitudinal modes, which are more directly associated with magnetization reversal. For spin resonance, however, it is the transverse modes, which are more directly related with magnetization precession and therefore with magnetization resonance. There are two main approaches, the so-called "statistical" approach and the "kinetic" approach.

In the statistical approach [26,27], which has a phenomenological character, the resonance conditions are evaluated by averaging over the equilibrium statistical distribution of all possible directions of particle magnetization moment. Typically, this model assumes that the magnetocrystalline anisotropy is smaller than the interaction energy between the magnetization and the applied field. While modeling can effectively represent some of the experimental results, the averaging of the magnetic anisotropy is not fully clear.

Another approach is also based on the Landau-Lifshitz equation of magnetization dynamics. In this model [26], $\boldsymbol{H}$ is changed by $\boldsymbol{H} \mathrm{l}_{\mathrm{oc}}$ to take into account both the external field and the effective field of the intrinsic anisotropy of the NPs. The dependence of the line width with temperature is expressed by 


$$
\Delta H=\Delta H_{T} L(X)
$$

where $\Delta H_{\mathrm{T}}$ is the limiting value of the line width for bulk particles and $L(X)$ is a Langevin type $(L(X)=\cot \mathrm{h} X-1 / X)$ of a phenomenological temperature factor $X$ (see Berger et al. [26] for more details).

Experimentally, the behavior of the line width and the resonance field of SPM phases of metallic and oxide NPs is quite clear; as temperature increases, the line width decreases and the resonance field increases, as illustrated by means of some recent results, see Figure 11 [23].

As temperature increases, the resonance field increases from $243 \mathrm{mT}$ for $102 \mathrm{~K}$ to $333 \mathrm{mT}$ for $473 \mathrm{~K}$. This is due to the fact that for temperatures below the Curie transition, there is an internal field formed by all the possible contribution to the ordered state:

$$
H_{\text {int }}=H_{\text {exch }}+H_{\text {anis }}+H_{\text {dem }}+\ldots
$$

where $H_{\text {exch }}$ is the exchange interaction, $H_{\text {anis }}$ is the anisotropy field, and $H_{\text {dem }}$ is the demagnetization field. As a result, the magnetization vector is subjected to a total field composed by the internal field and the applied field,

$$
H_{\mathrm{tot}}=H_{\mathrm{appl}}+H_{\mathrm{int}}
$$
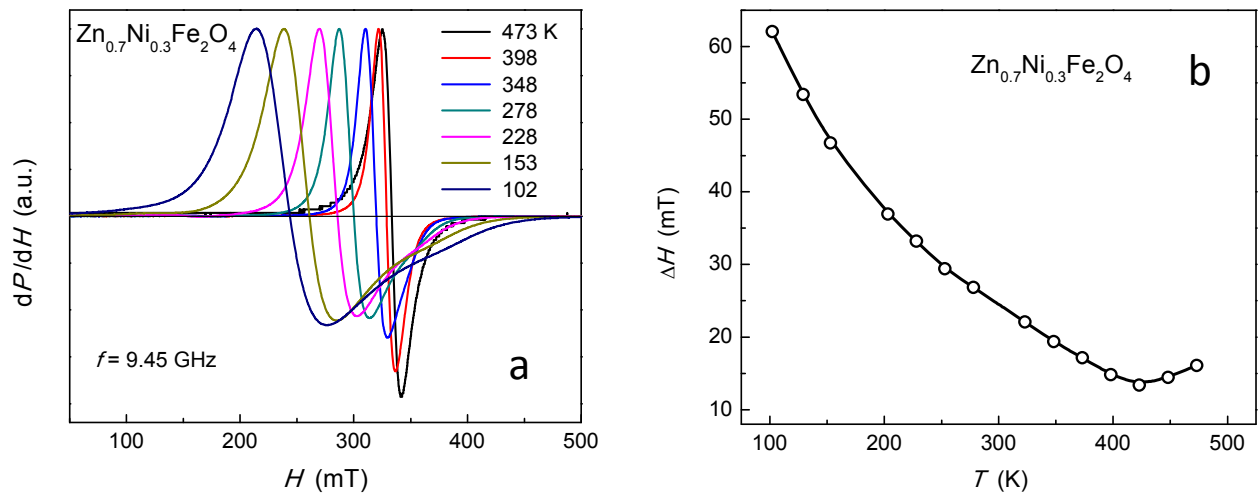

Figure 11. (a) ESR results on $\mathrm{Zn}_{0.7} \mathrm{Ni}_{0.3} \mathrm{Fe}_{2} \mathrm{O}_{4}$ ferrite NPs at a microwave frequency of $9.45 \mathrm{GHz}$, in the $102-473$ temperature range [22]. Signals were normalized to have the same value for the maximum (section of the resonance signal for $H<H_{\text {res }}$ ) to facilitate comparisons; (b) temperature behavior of the line width (peak to peak value) [23].

In order to fulfill the resonance conditions (Larmor equation), a smaller external field is required. In the paramagnetic state, thermal energy has overwhelmed the exchange coupling, and the resonance field is identical to the applied field. On the other hand, it is interesting to 
note again that in the SPM state, the exchange interaction is fully effective, and it remains active up to the Curie transition.

In SPM phases, $H_{\text {anis }}$ should be small and decreasing as $T$ increases and thermal energy progressively overwhelms it, but it certainly retains some influence, especially at temperatures close to $T_{\mathrm{B}}$. The transition from the ordered (ferrimagnetic) to the SPM phase is essentially continuous. This is more evident in the $H>H_{\text {res }}$ section of the resonance signal, as this section exhibits a larger broadening and becomes asymmetric. As we will see, this section of the signal is also associated with the magnetocrystalline anisotropy in the case of ferrimagnetic phases.
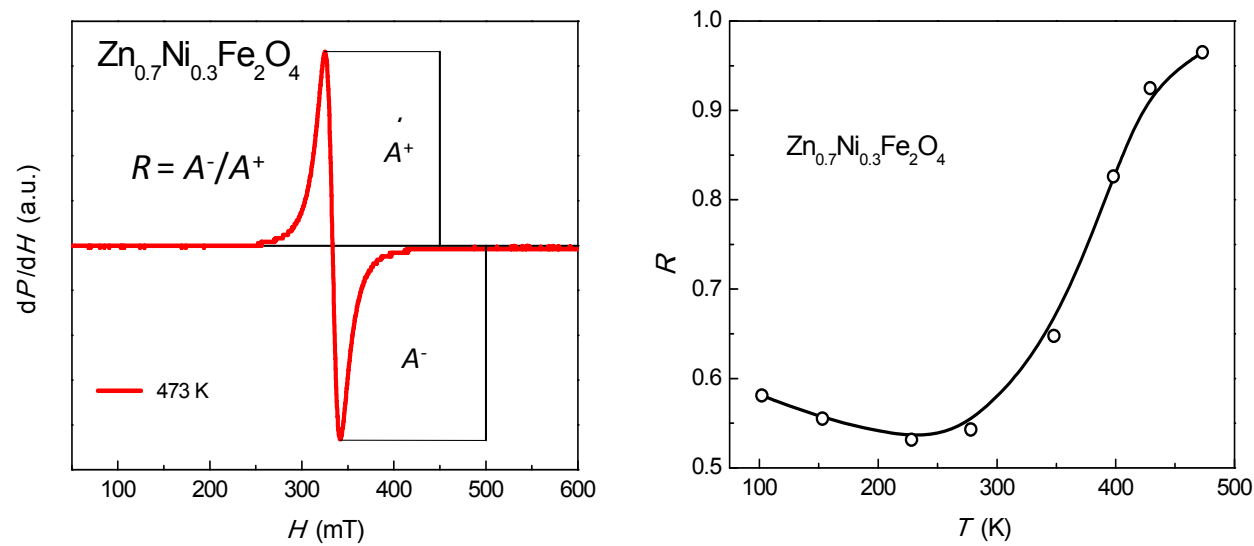

Figure 12. (a) Definition of the $R$ symmetry parameter, as the ratio of resonance signal amplitude $L_{\mathrm{A}}\left(H>H_{\text {res }}\right)$ to $L_{\mathrm{B}}(H$ $<H_{\text {res }}$; (b) behavior of $R$ for the measured temperature range [23].

As an attempt to get more insight into the changes associated with the SPM phase, we have proposed a parameter $R$, which measures the symmetry of the resonance signal. This parameter is defined as the ratio $R=A^{-} / A^{+}$, between the amplitude of the section in the negative part of the spectrum, $A^{-}$, and the amplitude of the positive part, $A^{+}$, as shown in Figure 12. In many ferrite NPs, $R$ has been observed to tend to unity as temperature increases and the ferrite progresses to the paramagnetic phase.

\section{Ferromagnetic resonance}

While the physical principals behind EPR experiments are due to the Zeeman effect, ferromagnetic resonance has a different description because FMR arises from the precessional motion of the whole magnetization $\mathbf{M}$ of a ferromagnetic material in an external magnetic field $\mathbf{H}$ as shown in Figure 13 [27]. 

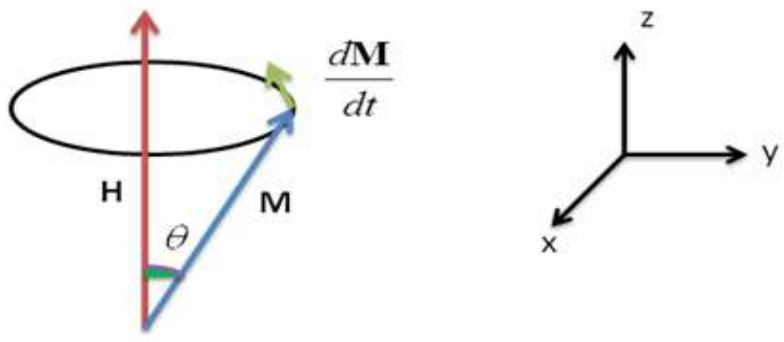

Figure 13. Magnetization vector $M$ precessing around an external field $H$.

Actually ferromagnetic resonance at microwave frequencies is similar in principle to nuclear spin resonance. The total electron magnetic moment of the sample precesses around the direction of the external field and the absorption is due to the energy absorbed from the $r f$ transverse field when its frequency is equal to the precessional frequency $[27,28]$.

The ferromagnetic resonance was unknowingly discovered by V. K. Arkad'yev when he observed the absorption of ultrahigh frequency radiation by ferromagnetic materials. However, it was officially observed by Griffiths in the Clarendon Laboratory in Oxford in 1946 and then confirmed by Yager and Bozorth who found a sharp peak in a supermalloy (Ni 75\%-Fe $20 \%-$ Mo 5\%) sample for a field strength near $5 \mathrm{kOe}$ with a 24,000-Mc/s frequency [28].

Ferromagnetic resonance was theoretically discussed before it was knowingly observed, particularly in the paper written by Landau and Lifshitz in Kharkov in 1935. C. Kittel provided a more complete theoretical formulation in 1951 [27,28]. The resonant absorption in ferromagnetic metals for a given frequency is controlled by an effective field $H_{\text {eff }}$ which is the sum of an external applied field and the contributions of internal magnetization, while in EPR the effective field in which the absorption takes place is the same as the external applied field [27-29].

From the theoretical point of view, the ferromagnetic resonance is described by the FMR equations. These are differential equations that connect time derivatives of the magnetization components with the components of the magnetization, the external field, the static magnetic susceptibility, and the time relaxation $[9,27]$. The FMR equations are different depending on the temperature regime which one is working in. Below the Curie temperature, the FMR equations are called the Landau-Lifshitz equations. Over the Curie temperature, the FMR equations lead to the Bloch-type ferromagnetic-resonance equations [9].

These equations were obtained empirically; however, for a better understanding of the microscopic phenomena, a quantum statistical derivation was developed by O. A. Olkhov and B. N. Provotorov, taking into account a system with spin magnetism placed in a magnetic field composed by a constant field $H_{0}$ (which causes the state of full saturation), and the alternating field $H_{1}[9]$.

The Hamiltonian for this derivation contemplates the potential energy $\mu$ H.S, the exchange interaction term, the dipolar interaction term, the lattice energy, and the spin-lattice interac- 
tion. For the quantum statistical derivation, the relationship between the relaxation time and the correlation time is very important. The correlation time $\omega_{\mathrm{C}}$ is much less than the relaxation time $\omega_{R}$. This means that the equilibrium in the spin system is established by the strong exchange interaction, while the relaxation is conditioned by the weak dipolar interaction, so the equilibrium of the system is established by the strong exchange interaction much more quickly than the relaxation process. This assumption is confirmed by experimental data on the inelastic magnetic scattering of neutrons [9].

The principal result of the theory is that the resonance condition is given by $[9,28]$

$$
\omega_{0}=g \frac{e}{2 m c}\left\{\left[H_{z}+\left(N_{y}-N_{z}\right) M_{z}\right] \times\left[H_{z}+\left(N_{x}-N_{z}\right) M_{z}\right]\right\}^{1 / 2}
$$

Instead of the Larmor condition, where $\omega_{0}$ is the frequency at resonance, $\gamma=g e / 2 m c$, is the magnetomechanical ratio for an electron spin, $H_{z}$ is the static magnetic field, $M_{z}$ is the component of the magnetization along the $z$ axis, and $N_{x, y, z}$ are the components of the demagnetization.

It is important to take into account that the resonance condition is closely related to the demagnetization field, and this depends on the shape of the sample. For this reason, there are some special cases for the resonance frequency, for instance, the plane, the sphere, and a long circular cylinder $[27,28]$.

Another important consideration is that the energy of ferromagnetic crystals depends in part on the anisotropy energy. The anisotropy energy has an effect in the resonance condition. In the case of a single crystal, the value of the magnetic field required for resonance at fixed frequency depends on the direction of the crystal axes relative to the shape axes of the sample.

In a polycrystalline sample, the absorption line will, in general, look broader than in a single crystal sample because the distribution in direction of the crystalline axes causes a distribution in the field strengths for resonance $[27,28]$.

It is convenient to consider the effect of the anisotropy energy as an equivalent magnetic field $H^{\text {s; }}$ this field is defined such that the torque exerted on the sample by this field is equal to the torque exerted by the anisotropy energy,

$$
\frac{\partial f}{\partial \theta}=\mathbf{M}_{S} \times \mathbf{H}^{s}
$$

However, this equivalent magnetic field is not completely determined by equation 2 because the magnitude and the direction are arbitrary. One can express the components of $\mathbf{H}^{\mathrm{S}}$ in terms of an effective demagnetization factor, which will be added to the usual demagnetization factor in the equation for the resonance frequency. The resonance condition depends on the shape of the sample as well as on the orientation of the crystal. 


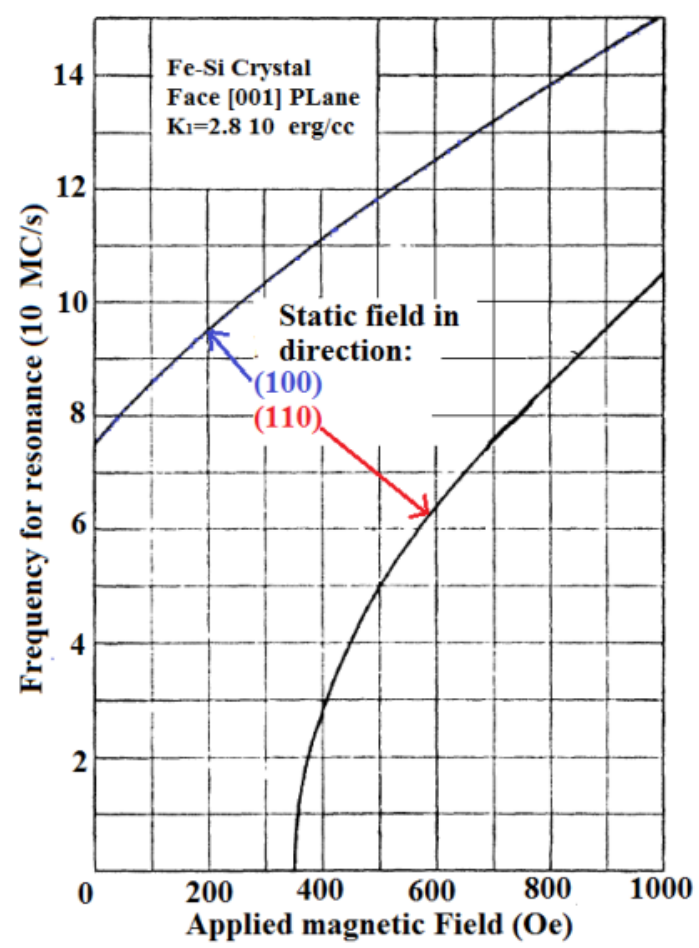

Figure 14. Comparison of the theoretical resonance condition for [100] and [110] directions in Fe-Si single crystal with a (001) plane surface. Adapted from Kittel [28].

A classic example of this is the difference of the magnetic field required for resonance with the (100) crystal face in a crystal with cubic anisotropy when the static field $H_{z}$ is in the [110] direction and when $H_{z}$ is in the [100], see Figure 14. In the first case, $H_{z}$ is greater than in the second. The difference is of the order of $4 \mathrm{~K}_{1} / \mathrm{M}_{\mathrm{S}}$.[6].

\subsection{Ferrimagnetic nanoparticles}

Nanoparticles of magnetic systems are of particular interest since the reduction to nanosized dimensions of the magnetic lattice gives rise to many interesting and different properties with respect to bulk materials. For instance, the surface spins, which constitute an important fraction of the total spins, undergo decrease in the coordination number and, therefore, a deficiency in exchange interactions. This situation can lead to severe changes in magnetization and anisotropy behavior. The relaxation processes of magnetization are also strongly temperature and size dependent.

In order to make a comparison between the three phases in ferrite NPs, Figure 15, we have selected the spectra obtained at $103 \mathrm{~K}$ (ordered, ferrimagnetic phase), $323 \mathrm{~K}$ (SPM phase), and $448 \mathrm{~K}$ (paramagnetic phases) [23]. The main difference exhibited by the ordered phase (in 
addition to the decrease in the resonance field) is the broadening in the section at $H>H_{\text {res }}$ i.e., $A^{-}$. The origin of this feature should be found in the effect of the internal field, which is the main difference with the other phases, and in particular, in the magnetocrystalline anisotropy field.

We present here two examples to show the complexity of changes driven by the reduction to nanosize dimensions, as illustrated by FMR. The first example involves cobalt-doped zinc ferrite nanoparticles $\left(\mathrm{Co}_{0.73 y} \mathrm{Zn}_{0.73(1-y)} \mathrm{Fe}_{2.18} \square_{0.09} \mathrm{O}_{4}\right)$. After synthesis, they were solubilized in aqueous solution containing $10 \%$ of polyvinyl alcohol [29]. Upon evaporation and during the polymerization process, the samples were subjected to a magnetic field in order to obtain an alignment of anisotropy axes.

The magnetization curves for these samples showed that the coercitivity increases as the percentage of Co increased, as well as the ratio of the remanence magnetization to saturated magnetization $M_{\mathrm{R}} / M_{\mathrm{S}}$. This latter result was interpreted as a change from uniaxial anisotropy for the $\mathrm{Zn}$ ferrite to a cubic anisotropy for the ferrites containing Co. This result was confirmed by the FMR spectrums as shown in Figure 15, associated with a broadening of the line width. This is attributed to the strong cubic magnetocrystalline anisotropy of cobalt ions in octahedral sites. This is somewhat contradictory as in bulk materials, it is well known that $\mathrm{Co}^{2+}$ on octahedral sites leads to change in the anisotropy sign (negative in most ferrites) from negative to positive (hence, uniaxial) [30].

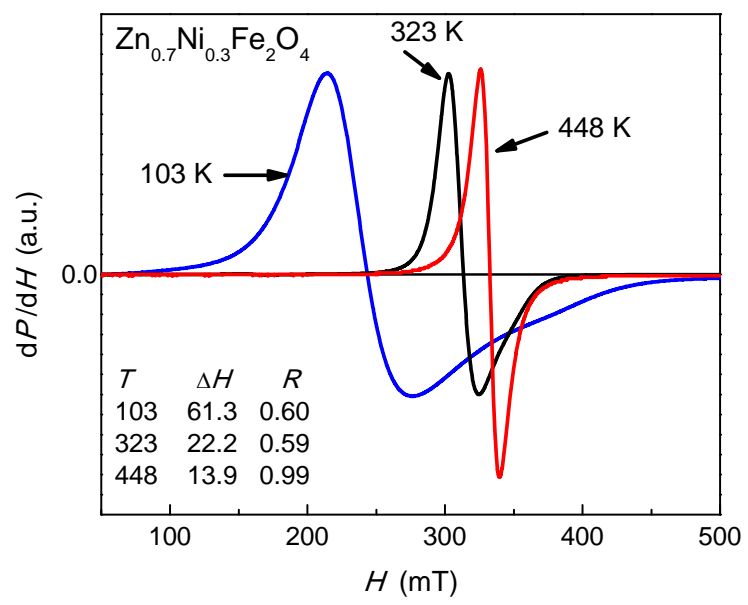

Figure 15. Typical signals associated with the three magnetic phases; paramagnetic at $448 \mathrm{~K}$, ferrimagnetic at $103 \mathrm{~K}$, and in the middle, the SPM phase which shows a progressive behavior from ferrimagnetic to paramagnetic as $T$ increases [23].

The FMR spectra of 3.7-nm nanoparticles show a drastic change when $10 \%$ of cobalt ions were added. A line width broadening and a shift in resonance toward lower fields are observed as 
the temperature decreases, and this behavior is more evident for the nanoparticles containing Co (Figure 16).

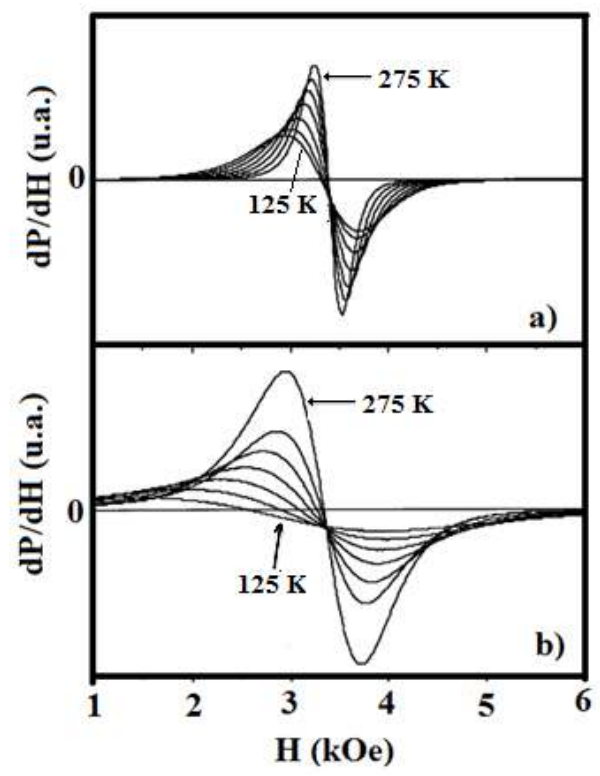

Figure 16. FMR spectra of 3.7-nm diameter ferrite nanoparticles dispersed in PVA, at various temperatures. (a) Zinc ferrite nanoparticles with no cobalt $(y=0)$. (b) Zinc ferrite with $y=10$ of cobalt. Adapted from Gazeau et al. [31].

The next example is a ferrite nanoparticle system of maghemite $\left(\gamma-\mathrm{Fe}_{3} \mathrm{O}_{2}\right)$ [31]. The oxide $\gamma$ $\mathrm{Fe}_{3} \mathrm{O}_{2}$ is an inverse spinel structure with all iron in the trivalent state and ion vacancies in the octahedral sublattice. Anisotropy properties studies have been done for $\gamma-\mathrm{Fe}_{3} \mathrm{O}_{2}$ nanoparticles of diameter from 4.8 to $10 \mathrm{~nm}$ dispersed in glycerol forming a magnetic fluid. This study is done by measuring the samples in a temperature range from $3.5 \mathrm{~K}$ to $300 \mathrm{~K}$ with (i) zero field cooled (ZFC) and thus randomly oriented anisotropy axes and (ii) field cooled (FC) with $H_{\mathrm{fr}}=$ $10 \mathrm{kOe}$. The FMR spectra showed a decrease in the resonance field, and a broadening of the line width, Figure 17. The parameters are shown in Figure 5 [31].

The resonance fields and the line widths of the $\gamma-\mathrm{Fe}_{3} \mathrm{O}_{2}$ samples are represented as a function of temperature, see Figure 18. The ZF experiments are done for $\theta=0^{\circ}$ and for $\theta=90^{\circ}$, where $\theta$ is the angle between the directions of the freezing field and the magnetizing field.

In the field cooled samples, the orientation distribution of the anisotropy axes results from the competition between the magnetic energy, the anisotropy energy and the thermal energy. The distribution in orientation of the anisotropy axes affects the FMR spectrum in angular variation and line width. On the other hand, there is also an effect of the particle size in the anisotropy. This is observed in Figure 19, where $H_{\text {res }}\left(90^{\circ}\right)-H_{\text {res }}\left(0^{\circ}\right)$ and $\Delta H$ are plotted as a function of temperature for different particle sizes. The observed reduction of $H_{\text {res }}\left(90^{\circ}\right)-H_{\text {res }}\left(0^{\circ}\right)$ is related 


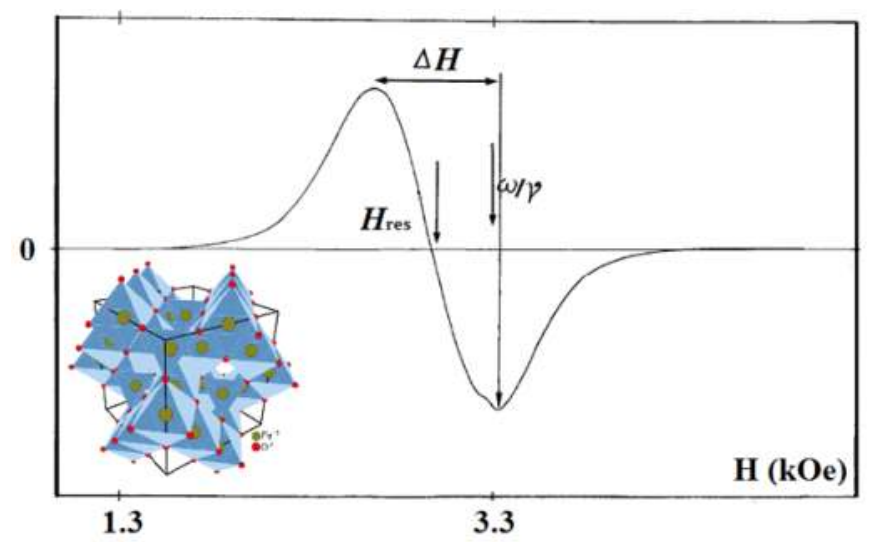

Figure 17. FMR spectrum of maghemite $\left(\gamma-\mathrm{Fe}_{3} \mathrm{O}_{2}\right)$ nanoparticles of $7 \mathrm{~nm}$ of average diameter at room temperature and microwave frequency $f=9.26 \mathrm{GHz}$. The inset shows the spinel structure of maghemite. Adapted from Gazeau et al. [31].
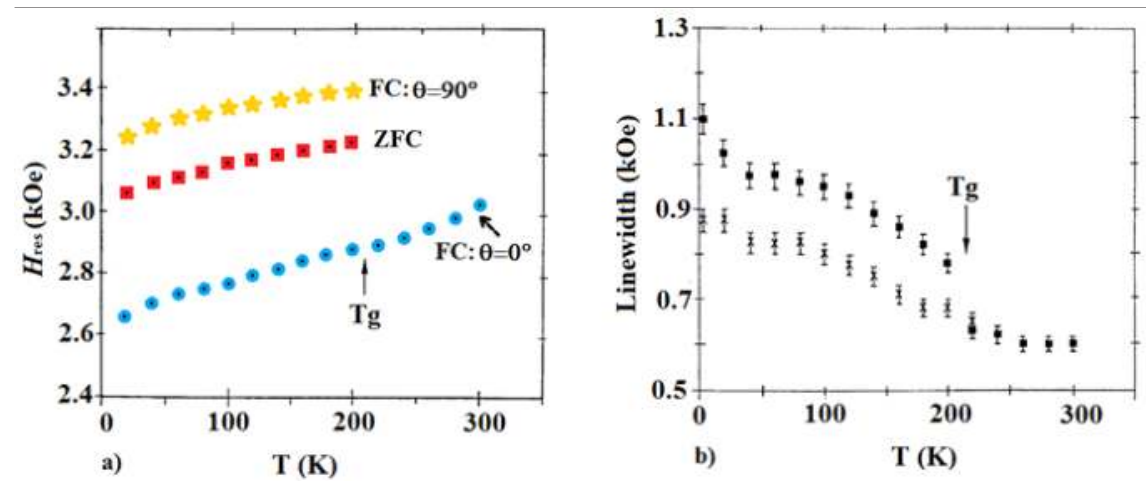

Figure 18. (a) Temperature dependence of the resonance field for 10-nm diameter $\gamma$ - $\mathrm{Fe}_{3} \mathrm{O}_{2}$ nanoparticles cooled under a $10-\mathrm{kOe}$ magnetic field (FC) and cooled without field (ZFC). The arrow indicates the melting temperature of the ferrofluid matrix. (b) Temperature dependence of the peak-to-peak line width for the same samples. Adapted from Gazeau et al. [31].

to the particle size decrease because of the orientation distribution of the anisotropy axes. The FMR study indicates that nanosized particles possess uniaxial anisotropy, even though bulk maghemite has a cubic anisotropy.

By using a theoretical model [32] based on the Landau-Lifshitz-Gilbert dynamics, it is possible to show that for a single domain assembly of magnetic NPs with randomly distributed anisotropy axes, a large broadening of the absorption line is obtained, see Figure 19. In addition to a shift of the resonance frequency, the distribution of anisotropy axes gives rise to a significant change in the shape and symmetry of the line. Figure 20 was calculated at zero temperature; as temperature increases, the shift in resonance field decreases, and due to the typical decrease in anisotropy energy with $T$, the line broadening is also reduced. 

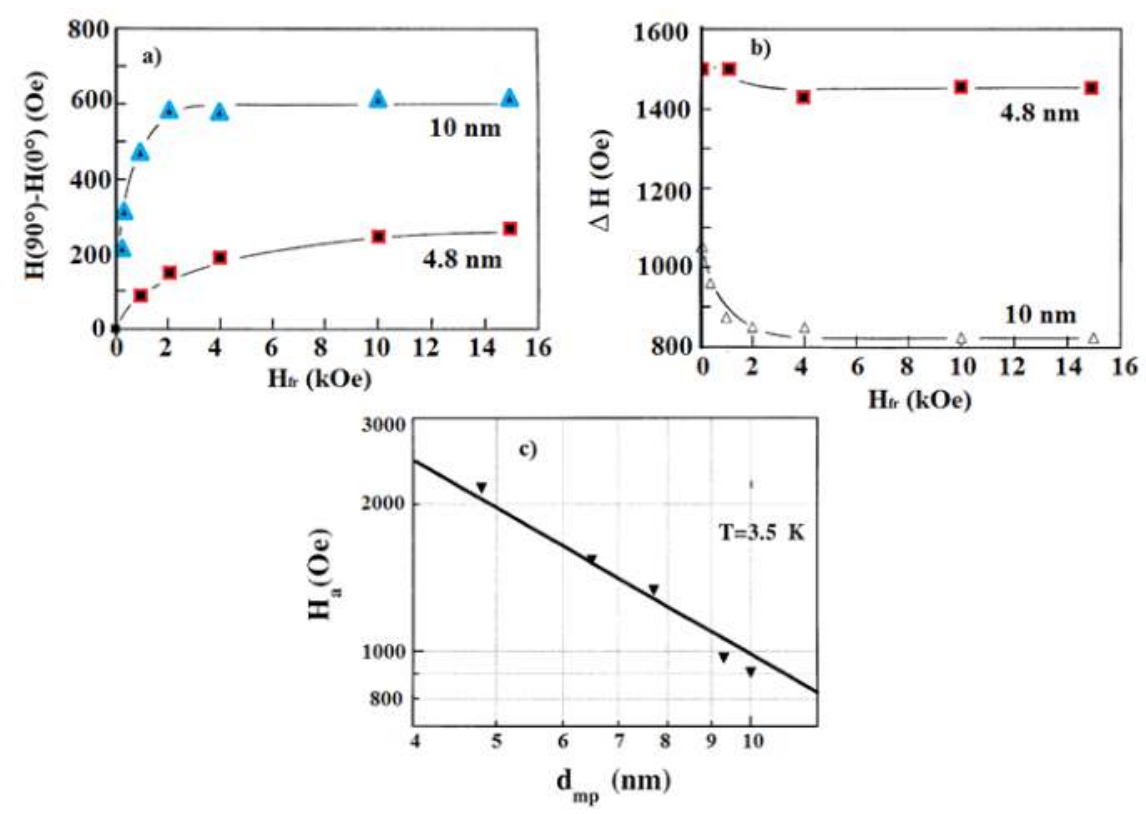

Figure 19. (a) Difference of the resonance fields for anisotropy axis orientated at $90^{\circ}$ and $0^{\circ}$ form the magnetic field. (b) Peak-to-peak line width as a function of microwave field for two different size of $\gamma-\mathrm{Fe}_{3} \mathrm{O}_{2}$ nanoparticles. (c) Anisotropy field deduced for angular variations at $3.5 \mathrm{~K}$ as a function a particle size. Adapted from Sukhov et al. [32].

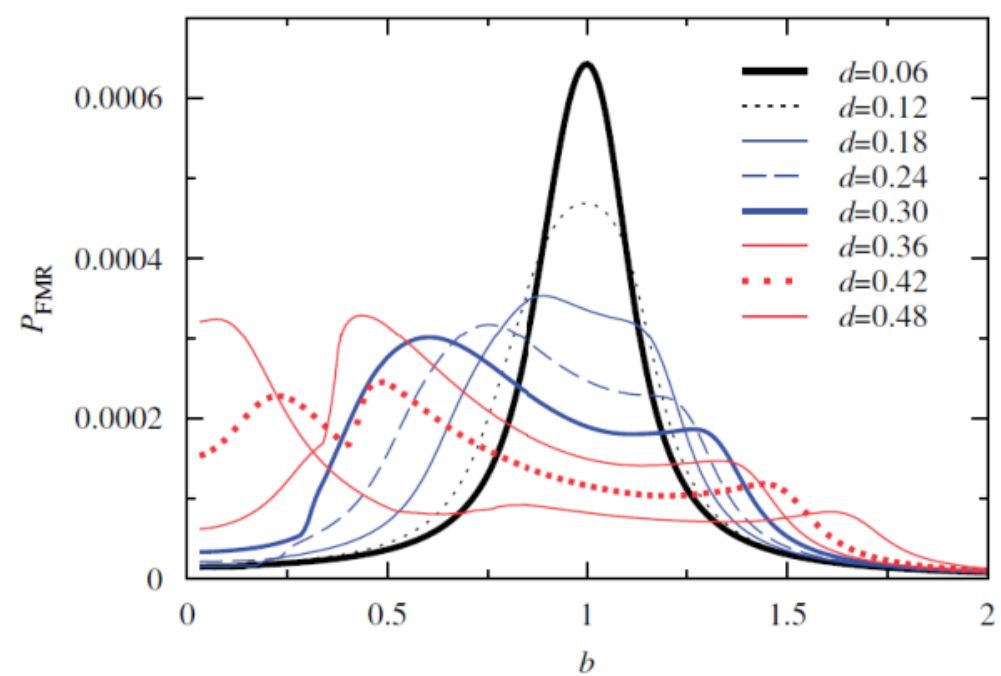

Figure 20. Absorbed FMR power (arbitrary units) vs reduced magnetic field at zero temperature and for different values of the damping parameter in the Landau-Lifshitz-Gilbert dynamics [32]. 


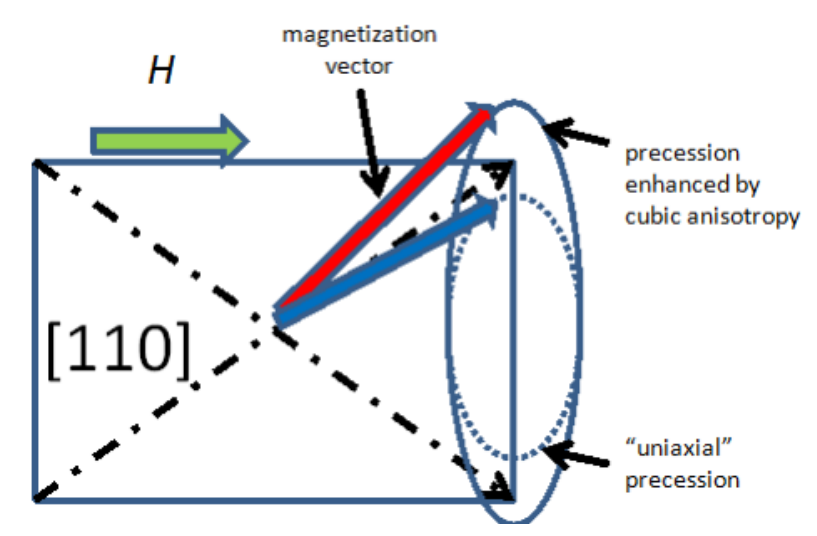

Figure 21. Interpretation of the line broadening in ferrites with cubic anisotropy. On a [110] crystal plane, containing two easy axis of the $<111>$ family, which have an influence on the magnetization dynamics, leading to an increase in the precession amplitude.

These results were obtained for the case of uniaxial materials; in the case of most ferrites with cubic anisotropy axes, it would be expected a larger broadening. In a very cartoon-like model, it is possible to imagine that the magnetization is first oriented along the applied field, which has a value closer to the anisotropy field. Once close to the saturation and once satisfied the resonance conditions, the magnetization is driven by the microwave radiation to precess uniformly, that is in phase. However, magnetocrystaline anisotropy is strong at these temperatures, and it is possible to consider that the magnetization vector can still feel its influence and precesses with a tendency toward the cubic axes. This dynamics leads to a broadening of the line, as far as the main result will be the combination of precession along several anisotropy axes, and has to be averaged over a large NPs population. Figure 21 illustrates this interpretation.

\section{Conclusions}

It can be established that systematic and significant differences occur in the resonance signal of ferrite NPs, associated with the magnetic structure. Paramagnetic phases lead to a high resonance field (typically in the $\sim 330 \mathrm{mT}$ range for the $X$ band) with a small line width and a tendency to a symmetric signal $(R \sim 1)$ (see signal at $448 \mathrm{~K}$ in Figure 15), in contrast with the ferromagnetic phase, which exhibits a lower resonance field, broad line width and asymmetric shape (signal at $103 \mathrm{~K}$, Figure 15). Between these two different behaviors, there is an SPM phase showing a resonance field closer to the one for the paramagnetic phase, a progressive decrease in the broadening of the minimum section of the line (absorption at $H>H_{\text {res }}$ ), and therefore an increase in the $R$ parameter toward 1.0. The exact transition temperatures are not easy to establish since evolution from one phase to the next is quite continuous. 
As a general conclusion, these reviews confirm that electron spin resonance is a very sensitive experimental method that can be used to characterize magnetic phases in the complex field of magnetic nanoparticles.

\section{Acknowledgements}

Authors acknowledge partial support from DGAPA-UNAM, Mexico, through grant PAPIIT IN 101412.

\section{Author details}

Rebeca Díaz-Pardo and Raúl Valenzuela*

*Address all correspondence to: monjaras@unam.mx

Institute for Materials Research, National Autonomous University of Mexico, Circuito

Exterior, Ciudad Universitaria, Coyoacán, México D.F., México

\section{References}

[1] Guimaraes A.P. Principles of Nanomagnetism. Berlin: Springer-Verlag; 2009.

[2] Valenzuela R. Magnetic Ceramics. Cambridge: Cambridge University Press; 2005.

[3] Bragg, W.H. "The structure of the spinel group of crystals." Philosophical Magazine $1915 ; 30,305-315$.

[4] Kittel C. Introduction to Solid State Physics. New York: John Wiley \& Sons Inc; 2005.

[5] Abragam A., Bleaney B. Electron Paramagnetic Resonance. Oxford: Oxford University Press; 2012.

[6] Weil John A., Bolton J.R. Electron paramagnetic resonance. New York: John Wiley \& Son; 2007.

[7] Junk M.J.N. Assessing the Functional Structure of Molecular Transporters by EPR Spectroscopy. Berlin: Springer; 2012.

[8] Feher G., Kip A.F. "Electron spin resonance absorption in metals. I. Experimental," Physical Review 1955; 98, 337-348.

[9] Olkhov O.A., Provotorov B.N. "Quantum statistical derivation of ferromagnetic-resonance equations," Physical Review 1965; 140, 1296-1303. 
[10] Koksharob Y.A., Pankratov D.A., Gubin S.P., Kosobudsky I.D., Beltran M., Khodorkovsky Y., Tishin A.M. "Electron paramagnetic resonance of ferrite nanoparticles," Journal of Applied Physics 2001; 89, 2293-2298.

[11] Dyson F.J. "Electron spin absorption in metals. II. Theory of electron diffusion and the skin effect," Physical Review 1955; 98, 349-359.

[12] Khabibullin B.M., Kharakhashýan E.G. "Conduction-electron paramagnetic resonance in metals," Soviet Physics Uspekhi 1974; 16, 806-818.

[13] Dalal N.S., Murillo C.A. "The usefulness of EPR spectroscopy in the study of compounds with metal-metal multiple bonds," Dalton Transactions 2014; 43, 8565-8576.

[14] Lindsay D.L. “Oxide minerals, short course notes," Mineralogical Society of America, Washington, DC, 1976; 3, L66-L71.

[15] Néel L. “Théorie du traînage magnétique des ferromagnétiques en grains fins avec applications aux terres cuites," Annales de Géophysique 1949; 5, 99-136.

[16] Brown W. Jr. "Thermal fluctuations of a single domain particle," Physical Review $1963 ; 130$ (5) 1677-1686.

[17] Wernsdorfer W., Bonet Orozco E., Hasselbach K., Benoit A., Barbara B., Demmoncy N., Loiseau A., Pascard, H., Mailly D. "Experimental evidence of the Néel-Brown model for magnetization reversal," Physical Review Letters 1997; 78, 1791-1795.

[18] Victora R.H. "Predicted time dependence of the switching field for magnetite," Physical Review Letters 1989; 63, 457-459.

[19] de Biasi E., Zysler R., Ramos C.A., Knobel M. "A new model to describe the crossover from superparamagnetic to blocked magnetic nanoparticles." Journal of Magnetism and Magnetic Materials 2008; 320, e312-e315.

[20] de Biasi R.S., Devezas T.C. “Anisotropy field of small magnetic particles as measured by resonance." Journal of Applied Physics 1978; 49, 2466-2469.

[21] Cruz-Franco B., Gaudisson T., Ammar S., Bolarin-Miró A.M., Sánchez de Jesus F., Mazaleyrat F., Nowak S., Vázquez-Victorio G., Ortega-Zempoalteca R., Valenzuela R. "Magnetic properties of nanostructured ferrites," IEEE Transactions on Magnetics 2014; 50(4) 280016-1-280016-5.

[22] Raikher Yu.L., Stepanov V.I., "Magnetization dynamics of single-domain particles by superparamagnetic theory," Journal of Magnetism and Magnetic Materials 2007; 316(2) 417-421.

[23] Raikher Y.L., Stepanov V.I. "Intrinsic magnetic resonance in superparamagnetic systems," Physical Review B 1995; 51 (22) 16428-16431.

[24] Flores-Arias Y., Vázquez-Victorio G., Ortega-Zempoalteca R., Acevedo-Salsa U., Ammar S., Valenzuela R. "Magnetic phase transitions in ferrite nanoparticles character- 
ized by electron spin resonance (ESR)." Journal of Applied Physics 2015; 117(17) 17A503.

[25] Coffey W.T. Krothers D.S.F., Kalmyvov Yu.P., Massawe E.S., Waldron J.T. Physical Review E 1949; 49, 1869-1872.

[26] Aharoni A. "Susceptibility resonance and magnetic viscosity." Physical Review B 1992; 46, 5434-5441.

[27] Berger R, Bissey J.C., Kliava J., Baubric H., Estournes C. “Temperature dependence of superparamagnetic resonance of iron oxide nanoparticles." Journal of Magnetism and Magnetic Materials (2001) 234, 535-44.

[28] Yulikov M.M., Purtov P.A. “FMR study of superparamagnetic Ni particles with weak and strong anisotropy." Applied Magnetic Resonance 2005; 29, 231-249.

[29] Kittel C. "Ferromagnetic Resonance," Le Journal de Physique et le Radium 1951; 12, 291-302.

[30] Hochepied J.F., Pileni M.P. “Ferromagnetic resonance of nonstoichiometric zinc ferrite and cobalt-doped zinc ferrite nanoparticles." Journal of Magnetism and Magnetic Materials 2001; 231, 45-50.

[31] Bickford L.R., Pappis J., Stull J.L. "Magnetostriction and permeability of magnetite and cobalt-substituted magnetite." Physical Review 1955; 99, 1210-1214.

[32] Gazeau F., Bacri J.C, Gendron F., Perzynski R., Raikher Yu.L., Stepanov V.I., Dubois E. "Magnetic resonance of ferrite nanoparticles: evidence of surface effects." Journal of Magnetism and Magnetic Materials 1998; 186, 175-187.

[33] Sukhov A., Usadel K.D., Nowak U. "Ferromagnetic resonance in an ensemble of nanoparticles with randomly distributed anisotropy axes." Journal of Magnetism and Magnetic Materials 2008; 320, 31-35. 
